\title{
Mussel-Inspired Surface-Imprinted Sensors for Potentiometric Label- Free Detection of Biological Species
}

\author{
Rongning Liang, Jiawang Ding, Shengshuai Gao, and Wei Qin*
}

\begin{abstract}
Using sensors to quantify clinically relevant biological species has emerged as a fascinating research field due to their potential to revolutionize clinical diagnosis and therapeutic monitoring. Taking advantage of the wide utility in clinical analysis and low cost of potentiometric ion sensors, we demonstrate a method to use such ion sensors to quantify bioanalytes without chemical labels. This is achieved by combination of chronopotentiometry with a mussel-inspired surface imprinting technique. The biomimetic sensing method is based on a blocking mechanism by which the recognition reaction between the surface imprinted polymer and a bioanalyte can block the current-induced ion transfer of an indicator ion, thus causing a potential change. The present method offers high sensitivity and excellent selectivity for detection of biological analytes. As models, trypsin and yeast cells can be

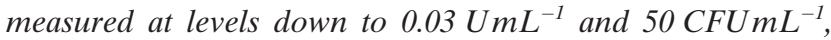
respectively.
\end{abstract}

In In recent years, reliable, efficient, and convenient analysis of biological analytes such as DNA, RNA, proteins, and even whole cells have attracted significant interest because of their great importance for many areas of healthcare and the life sciences ranging from clinical diagnosis and therapy to discovery and screening of new drug molecules. ${ }^{[1,2]}$ Current methods for quantitative detection of bioanalytes such as biomacromolecules (for example, DNA and proteins) still mostly rely on conventional methods including enzymelinked immunosorbent assays, ${ }^{[3]}$ biochemical tests, ${ }^{[4]}$ and polymerase chain reactions, ${ }^{[5]}$ while for larger bioanalytes (that is, whole cells), classical cell-culture-based assays are indispensable. Unfortunately, these well-established approaches have obvious drawbacks of complicated instrumentation, intensive labor, and time-consuming procedures. Especially, these tests cannot easily be performed in urban areas in developed countries as well as rural areas in developing countries because of their high cost.

Nowadays, the design of sensor platforms for rapid, onsite and low-cost detection of a variety of bioanalytes is exhibiting a great potential to dramatically change this situation. ${ }^{[6]}$ As one of the most commonly used electro-

[*] Dr. R. Liang, Dr. J. Ding, S. Gao, Prof. Dr. W. Qin

Key Laboratory of Coastal Environmental Processes and Ecological Remediation, Yantai Institute of Coastal Zone Research (YIC), Chinese Academy of Sciences (CAS); Shandong Provincial Key Laboratory of Coastal Environmental Processes, YICCAS Yantai, Shandong 264003 (P.R. China)

E-mail:wqin@yic.ac.cn

(2) Supporting information for this article can be found under: https://doi.org/10.1002/anie.201701892. chemical devices, polymeric membrane ion-selective electrodes (ISEs), have been widely applied in clinical diagnosis and environmental monitoring. ${ }^{[7]}$ Many clinical analyzers based on ISEs have been developed and are commercially available for monitoring analytes in clinical laboratories and at the bedside of critically ill patients. It can be estimated that each year over a billion measurements with ISEs are preformed throughout the world. ${ }^{[8]}$ Compared with other methods, ISEs are extremely inexpensive and only minimally perturb the samples. In particular, recent improvements in the detection limits of these sensors yielded sensors for the direct measurement in the subnanomolar concentration range. ${ }^{[9]}$ Such major achievements have facilitated new applications for which potentiometric sensors have not been used traditionally. ${ }^{[10]}$ These characteristics make these sensors particularly suitable for their use in bioanalyte detection. However, it should be noted that to date it is still a big challenge to carry out specific and direct potentiometric sensing of bioanalytes without chemical labeling since the receptor-bioanalyte interaction usually does not yield a measurable potential signal. ${ }^{[11]}$

Over the past decades, several potentiometric sensors have been explored for determination of biological analytes. The polymer membrane polyion sensors based on a nonequilibrium response mechanism can be used for direct and sensitive detection of biological species. ${ }^{[12]}$ However, the analytes are limited to polyions such as protamine and heparin, which can be readily extracted into the organic sensing membrane phase from the aqueous phase. Although potentiometric biosensors have been successfully used for ultrasensitive indirect measurements of proteins or bacteria, ${ }^{[10 a, b, 11,13]}$ these methods involve the complicated screening processes of antibodies or aptamers for bioanalytes. In particular, in these methods, chemical labels such as enzyme tags, redox tracers, and nanoparticle labels are often required. Clearly, a general approach for potentiometric sensing of a broad range of bioanalytes without chemical labels is highly desired.

Herein, we introduce a general, facile, and highly selective strategy for the label-free potentiometric sensing of bioanalytes. Molecularly imprinted polymers (MIPs) have been regarded as general materials for the selective recognition of a wide range of analytes with affinities and selectivities comparable to natural receptors such as antibodies and enzymes. ${ }^{[14,15]}$ In particular, they are less costly, easier to synthesize, and much more stable in harsh chemical environments compared to the natural receptors. However, the imprinting of bioanalytes still remains a challenge since traditional MIPs are highly cross-linked, making it difficult for bioanalytes to reach the binding sites buried in the 
interiors of the MIPs. ${ }^{[16]}$ The surface imprinting technique offers a unique protocol for bioanalyte imprinting compared to the traditional imprinting method. It exhibits excellent features such as high selectivity, accessible binding sites, and fast association/dissociation kinetics since the templateimprinting sites are situated at the surface or in the proximity of the polymer's surface. ${ }^{[17]}$ In this strategy, the proposed protocol is accomplished by combination of potentiometry with the novel surface imprinting technique. It is based on the blocking principle ${ }^{[18]}$ in which the recognition reaction between the surface imprinting polymer and a bioanalyte occurs at the surface of a polymeric membrane potentiometric sensor, thus blocking the flux of an indicator ion to the membrane. The induced potential change can be used as the analytical signal to quantitatively determine the concentration of the bioanalyte present in the sample. Note that, prior relevant work has mainly been done on either nonspecific membrane coatings containing surfactants ${ }^{[18 a]}$ and polyelectrolytes $^{[18 b]}$ or special biorecognition layers based on biotinavidin chemistry. ${ }^{[18 c, d]}$

Figure 1 illustrates the sensing mechanism of the proposed protocol. The sensor platform is composed of two layers: The surface recognition layer for the bioanalyte and the potentiometric transduction layer for the indicator ion. The former is fabricated by the surface molecular imprinting in the presence of the bioanalyte on the surface of the latter. After removal of the analyte molecules, the analyte-imprinted sites can be created. When the electrode comes into contact with the analyte solution, the recognition event at surface-imprinted layer blocks the ion transfer of the indicator ion through the recognition layer into the transduction layer, which results in a potential change. Based on the above mechanism, the measurements of biological analytes were achieved using the following procedures: 1) The potentiometric sensor modified with the surface imprinted layer was incubated with the sample solution for a certain time; 2) then the sensor was rinsed thoroughly with deionized water to avoid the nonspecific adsorption and transferred to a separate electrochemical cell containing the indicator ion; 3) finally, potentiometric detection was carried out by using the sensor with the accumulated analyte in the presence of the indicator ion.

In the present work, trypsin was chosen as the model bioanalyte in view of its great importance to human health. A versatile surface imprinting approach based on a marinemussel-inspired polydopamine (PDA) coating was employed

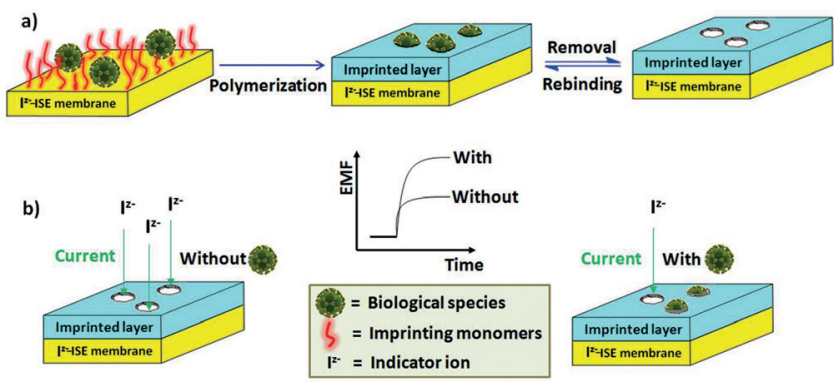

Figure 1. Schematic of a) the construction of mussel-inspired surfaceimprinted-layer-modified potentiometric sensor and b) chronopotentiometric detection of a bioanalyte. for trypsin imprinting. ${ }^{[19,20]}$ The nonimprinted polymer (NIP) was synthesized by a similar procedure in the absence of the template molecules. The surface morphology of the musselinspired imprinted layer attached onto the sensor surface was characterized by using scanning electron microscopy (SEM), and the top-view images are illustrated in Figure S1 in the Supporting Information. In contrast to the bare polymeric membrane (Figure S1 a), the image of the MIP-layer-covered membrane (Figure S1b) clearly exhibits a rough and porous but uniform surface. Atomic force microscopy (AFM) further confirms that the uniform imprinting layer was successfully deposited onto the sensor surface (Supporting Information, Figure S2). In addition, the average thickness of the PDA imprinting layer measured by a surface profilometer was found to be $484 \mathrm{~nm}$. Such a thin layer has the potential to overcome the mass-transfer limitation and nonquantitative recovery of the template from the imprinted polymer synthesized by the traditional methods, such as bulk polymerization, which can result in MIP particles or thick layers. ${ }^{[21]}$

Since using zero-current potentiometry to probe ion fluxes across the polymeric sensing membrane has met with only limited success, ${ }^{[18 \mathrm{~d}]}$ pulsed galvanostatic chronopotentiometry, which can rapidly and accurately control the ion extraction process, ${ }^{[22]}$ was applied to directly monitor the blocking effect induced by the trypsin recognition in the mussel-inspired surface imprinted layer. It was shown that larger indicators could cause larger flux changes on the membrane surface and hence induce larger signals (see below). Therefore, the polyion heparin was selected as the indicator ion (Figure 2a). For monitoring of the change in heparin flux, a fully reversible pulstrode polyanion sensor based on an ion-exchanger-free polymeric sensing membrane formulated with the lipophilic neutral salt tridodecylmethylammonium-dinonylnaphthalene sulfonate was constructed. ${ }^{[23]}$ An anodic galvanostatic current pulse is imposed across the polymeric membrane, which causes the heparin flux from the sample to the sensing membrane through the imprinted layer. The marco-command-controlled procedures for switching between the potentiostatic and galvanostatic steps are illustrated in the Supporting Information, Figure S3, as described previously. ${ }^{[23,24]}$ The pulse sequence was used as follows: 1) A zero current pulse with a duration of $1 \mathrm{~s}$ (open circuit), 2) a $5 \mu \mathrm{A}$ galvanostatic anodic current pulse of $1 \mathrm{~s}$ for the generation of an ion flux in the direction of the membrane, and 3) a potentiostatic potential pulse of $20 \mathrm{~s}$ for the regeneration of the membrane. ${ }^{[22]}$

Since many experimental factors can affect the performance of the above trypsin sensing system, parameters including molecule size and the concentration of the indicator ion (see Figures S4 and S5 in the Supporting Information, respectively), the applied anodic current amplitude and duration time (Figures S6 and S7, respectively), the surface imprinting time (Figure S8), and the concentration of acetic acid in the washing solution (Figure S9) were optimized.

Under the optimized conditions, the chronopotentiometric detection of trypsin based on the mussel-inspired surface imprinting technique using heparin as the indicator ion was performed. Figure $2 \mathrm{~b}$ displays the observed potential-time 
(a)
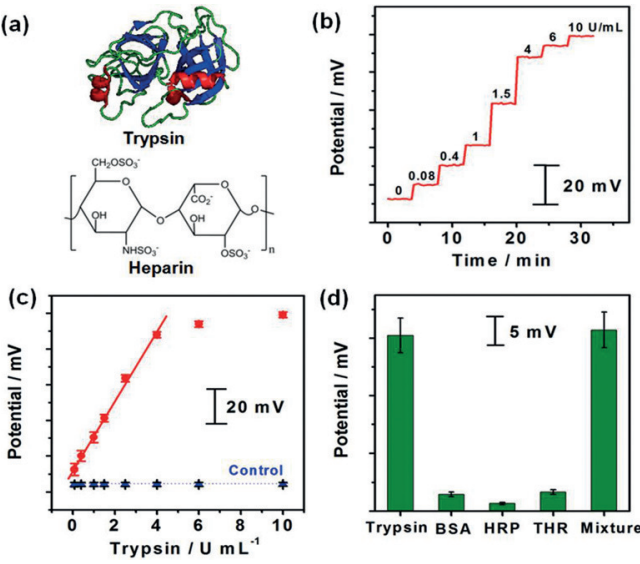

Figure 2. a) Crystal structure of the analyte trypsin and chemical structure of the indicator ion heparin. b) Observed time-dependent responses of the pulstrode chronopotentiometric sensor based on mussel-inspired surface imprinting in the presence of $40 \mathrm{U} \mathrm{mL}^{-1}$ heparin in a background of $10 \mathrm{~mm} \mathrm{NaCl}$ buffered at $\mathrm{pH} 7.4$ with a $0.01 \mathrm{~m}$ phosphate buffer solution (PBS) after incubations with trypsin at different concentrations for 10 min. Each step consists of 11 data points sampled at a $22 \mathrm{~s}$ interval at the end of $1 \mathrm{~s}$ of the $5 \mu \mathrm{A}$ galvanstatic current pulse. c) Corresponding calibration curve of the proposed sensor from the potential-time trace shown in (b). The dashed line corresponds to the control test of NIP. d) Pulstrode responses to trypsin, BSA, HRP, THR, and the mixture of these four proteins. The concentrations of trypsin and the other proteins were 1 and $100 \mathrm{U} \mathrm{mL}^{-1}$, respectively. Error bars represent the standard deviation for one electrode with three measurements.

trace of the pulstrode sensor to varying concentrations of trypsin with a measuring range of $0.08-10 \mathrm{U} \mathrm{mL}^{-1}$. Figure $2 \mathrm{c}$ shows the corresponding calibration curve of the pulstrode sensor from the potential-time trace. Each step in Figure $2 \mathrm{~b}$ consists of 11 data points sampled at a $22 \mathrm{~s}$ interval at the end of $1 \mathrm{~s}$ of the $5 \mu \mathrm{A}$ galvanostatic current pulse. As expected, the potential responses to heparin are indeed altered by the surface recognition reaction between the surface imprinted layer and trypsin. These results indicate that the instrumentally controlled ion flux of heparin can be partially blocked by the trypsin recognition event in the surface imprinted layer and thereby confirm that the indicator ion blocking effect induced by trypsin recognition can be used to quantitatively determine the concentration of trypsin. In addition, the change in potential as a function of the concentration of trypsin in the sample solution was found to be linear in the range of $0.08-4 \mathrm{U} \mathrm{mL}^{-1}$. In this case, a low detection limit $(3 \sigma)$ of $0.03 \mathrm{U} \mathrm{mL}^{-1}(n=7)$ could be obtained, which is at least one order of magnitude lower than those reported by other potentiometric methods. ${ }^{[25]}$ A series of five repetitive measurements of $1 \mathrm{UmL}^{-1}$ trypsin was utilized for evaluating the detection precision. This series yielded reproducible chronopotentiometric signals with a relative standard deviation of $3.7 \%$ (Supporting Information, Figure S10). Control experiments were also performed using a NIP coating as the surface recognition element (the dashed line in Figure $2 \mathrm{c}$ ). Compared with the potential responses of MIP-based sensor, no obvious potential changes can be observed for the NIP-based sensor, which further confirms that the potential signals are exclusively caused by the specific recognition interactions between the MIP binding sites in the surface imprinted layer and the target biomolecules.

The specificity of the proposed potentiometric sensor based on the mussel-inspired surface imprinting technique was evaluated by using other proteins including bovine serum albumin (BSA), horseradish peroxidase (HRP), and thrombin (THR). The concentration of trypsin was 100-fold lower than that of other three proteins. As shown in Figure $2 d$, the potential responses to BSA, HRP, and THR are rather negligible as compared to that to trypsin. Additionally, no obvious difference between the response to trypsin and that to the mixture of these four proteins can be observed. These results suggest that the proposed method offers an excellent selectivity. Such selectivity is presumably due to the strong hydrogen-bonding interactions between the amino and hydroxy groups of PDA and the carboxyl and amino groups of trypsin. In addition, the spatial shape matching between the well-fabricated imprinting cavities and the target molecules may also play an important role in trypsin recognition. ${ }^{[21]}$ The proposed sensor was finally applied to determination of trypsin in human urine samples with satisfactory results (Supporting Information, Table S1). These data clearly show that our strategy is effective for sensitive, selective, and rapid electrochemical detection of proteins.

To demonstrate the general applicability of the proposed strategy, a larger, more complex, and organized bioanalyte, yeast cells with a diameter of circa $4 \mu \mathrm{m}$, was selected as another model (Figure 3a). The chronopotentiometric responses to yeast cells were recorded over a concentration range of $100-5000 \mathrm{CFU} \mathrm{mL}^{-1}$ target yeast. The resulting pulstrode responses and corresponding calibration plot are shown in Figure 3 b,c. As illustrated, the diffusion of the indicator ion heparin can be inhibited by the recognition of yeast cells. The proposed sensor exhibits a well-defined concentration dependence suitable for yeast cell analysis with a linear response in the concentration range of 100-
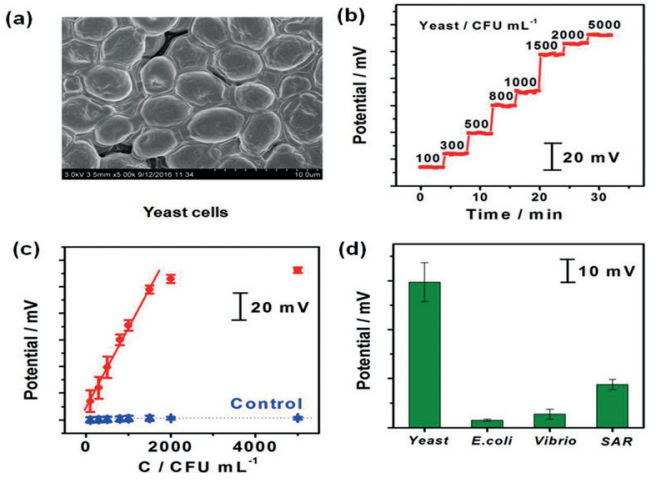

Figure 3. a) SEM image of yeast cells. b) Pulsed chronopotentiometric responses of the mussel-inspired surface-imprinted-layer-modified sensor to varying concentrations of yeast cells. c) Corresponding calibration plots. The dashed line shows the calibration curve for control experiments. d) Selectivity of the proposed sensor towards yeast over Escherichia coli (E. coli), Vibrio alginolyticus and Staphylococcus aureus (SAR): $500 \mathrm{CFU} \mathrm{mL}^{-1}$ of yeast cells; $5000 \mathrm{CFU} \mathrm{mL}^{-1}$ of other bacteria. Error bars represent the standard deviation for one electrode with three parallel measurements. Other conditions are as given in Figure $2 \mathrm{~b}$. 
$1500 \mathrm{CFU} \mathrm{mL} \mathrm{mL}^{-1}$ and a detection limit of $50 \mathrm{CFU} \mathrm{mL}^{-1}(3 \sigma, n=$ 7). This detection limit is much lower than those of PCRbased conventional methods. ${ }^{[26]}$ In contrast, control experiments were also carried out to confirm that the observed potential changes are due to the specific capture of yeast cells to the MIP binding sites in the surface layer (Figure $3 \mathrm{c}$ ). To demonstrate the specificity, three bacteria were chosen as the potential interferents including rod-shaped Vibrio alginolyticus and Escherichia coli (E. coli) and spherical Staphylococcus aureus (SAR). As shown in Figure $3 \mathrm{~d}$, the proposed sensor exhibits an excellent selectivity towards the spherical yeast cells over other bacteria even at higher concentrations (see the Supporting Information). This successful electrochemical determination of the much larger yeast cells demonstrates that our proposed method is compatible with different biospecies targets, indicating the high generality of the method.

In summary, this study demonstrates that biological analytes such as proteins and cells can be rapidly, sensitively, and selectively detected without chemical labeling by using the low-cost polymeric membrane ISEs, which have been extensively used in clinical analysis. The proposed approach is based on the blocking mechanism in which the recognition reaction between the mussel-inspired surface imprinted polymer and bioanalytes blocks the flux of the indicator ion from the sample solution to the sensing membrane. The proposed biomimetic sensing platform may have the potential to quantify many other targets such as DNA or virus, even tissues, through the use of suitable surface MIPs. This may pave the way to using ISEs to detect and quantify a variety of biological targets in clinical and environmental applications.

\section{Acknowledgements}

The authors thank the National Natural Science Foundation of China $(21475148,41576106,41206087)$, the Science and Technology Project of Yantai (2014ZH086), the Youth Innovation Promotion Association of CAS (2014190), and the Taishan Scholar Program of Shandong Province (TS20081159) for financial support of this study.

\section{Conflict of interest}

The authors declare no conflict of interest.

Keywords: biological species · biosensors - imprinting . mussel-inspired polymers $\cdot$ potentiometry

How to cite: Angew. Chem. Int. Ed. 2017, 56, 6833-6837 Angew. Chem. 2017, 129, 6937-6941

[1] a) A. G. Palmer, Chem. Rev. 2004, 104, 3623-3640; b) M. J. Lohse, D. Calebiro, Nature 2013, 495, 457-458; c) A. L. Bole, P. Manesiotis, Adv. Mater. 2016, 28, 5349-5366.

[2] a) D. R. Walt, Science 2000, 287, 451-452; b) E. Hendry, T. Carpy, J. Johnston, M. Popland, R. V. Mikhaylovskiy, A. J. Lapthorn, S. M. Kelly, L. D. Barron, N. Gadegaard, M. Kadodwala, Nat. Nanotechnol. 2010, 5, 783-787.
[3] B. Berg, B. Cortazar, D. Tseng, H. Ozkan, S. Feng, Q. Wei, R. Y. L. Chan, J. Burbano, Q. Farooqui, M. Lewinski, D. D. Carlo, Q. B. Garner, A. Ozcan, ACS Nano 2015, 9, 7857-7866.

[4] M. Doret, R. Cartier, J. Miribel, J. Massardier, M. Massoud, A. Bordes, S. Moret, P. Gaucherand, Clin. Biochem. 2013, 46, 18161819.

[5] L. Wu, L. L. van den Hoogen, H. Slater, P. G. T. Walker, A. C. Ghani, C. J. Drakeley, L. C. Okell, Nature 2015, 528, S86-S93.

[6] a) Y. Xiang, Y. Lu, Nat. Chem. 2011, 3, 697-703; b) F. Xia, X. Zuo, R. Yang, Y. Xiao, D. Kang, A. Vallée-Bélisle, X. Gong, J. D. Yuen, B. B. Y. Hsu, A. J. Heeger, K. W. Plaxco, Proc. Natl. Acad. Sci. USA 2010, 107, 10837-10841; c) S. S. Mahshid, S. Camiré, F. Ricci, A. Vallée-Bélisle, J. Am. Chem. Soc. 2015, 137, 1559615599.

[7] a) P. Bühlmann, E. Pretsch, E. Bakker, Chem. Rev. 1998, 98, 1593 -1688; b) E. Bakker, Anal. Chem. 2016, 88, 395-413; c) G. Jágerszki, Á. Takács, I. Bitter, R. E. Gyurcsányi, Angew. Chem. Int. Ed. 2011, 50, 1656-1659; Angew. Chem. 2011, 123, 16941697.

[8] J. Hu, A. Stein, P. Bühlmann, Angew. Chem. Int. Ed. 2016, 55, 7544-7547; Angew. Chem. 2016, 128, 7670-7673.

[9] T. Sokalski, A. Ceresa, T. Zwickl, E. Pretsch, J. Am. Chem. Soc. 1997, 119, 11347-11348.

[10] a) K. Y. Chumbimuni-Torres, Z. Dai, N. Rubinova, Y. Xiang, E. Pretsch, J. Wang, E. Bakker, J. Am. Chem. Soc. 2006, 128, 13676-13677; b) A. Numnuam, K. Y. Chumbimuni-Torres, Y. Xiang, R. Bash, P. Thavarungkul, P. Kanatharana, E. Pretsch, J. Wang, E. Bakker, J. Am. Chem. Soc. 2008, 130, 410-411; c) G. A. Crespo, G. Mistlberger, E. Bakker, J. Am. Chem. Soc. 2012, 134, 205-207; d) M. G. Afshar, G. A. Crespo, E. Bakker, Angew. Chem. Int. Ed. 2015, 54, 8110-8113; Angew. Chem. 2015, 127, 8228-8231.

[11] G. A. Zelada-Guillén, J. Riu, A. Düzgün, F. X. Rius, Angew. Chem. Int. Ed. 2009, 48, 7334-7337; Angew. Chem. 2009, 121, $7470-7473$.

[12] a) B. Fu, E. Bakker, J. H. Yun, V. C. Yang, M. E. Meyerhoff, Anal. Chem. 1994, 66, 2250-2259; b) Q. S. Ye, M. E. Meyerhoff, Anal. Chem. 2001, 73, 332-336; c) L. Wang, S. Buchanan, M. E. Meyerhoff, Anal. Chem. 2008, 80, 9845-9847.

[13] a) J. W. Ding, Y. Chen, X. W. Wang, W. Qin, Anal. Chem. 2012, 84, 2055-2061; b) J. W. Ding, J. H. Lei, X. Ma, J. Gong, W. Qin, Anal. Chem. 2014, 86, 9412 -9416; c) J. W. Ding, Y. Gu, F. Li, H. Zhang, W. Qin, Anal. Chem. 2015, 87, 6465-6469.

[14] a) R. N. Liang, D. A. Song, R. M. Zhang, W. Qin, Angew. Chem. Int. Ed. 2010, 49, 2556-2559; Angew. Chem. 2010, 122, 26102613; b) R. N. Liang, L. S. Chen, W. Qin, Sci. Rep. 2015, 5, 12462.

[15] a) K. Haupt, K. Mosbach, Chem. Rev. 2000, 100, 2495-2504; b) G. Wulff, Chem. Rev. 2002, 102, 1-28; c) D. Cai, L. Ren, H. Zhao, C. Xu, L. Zhang, Y. Yu, H. Wang, Y. Lan, M. F. Roberts, J. H. Chuang, M. J. Naughton, Z. Ren, T. C. Chiles, Nat. Nanotechnol. 2010, 5, 597-601.

[16] a) M. J. Whitcombe, I. Chianella, L. Larcombe, S. A. Piletsky, J. Noble, R. Porter, A. Horgan, Chem. Soc. Rev. 2011, 40, $1547-$ 1571; b) X. Shen, J. S. Bonde, T. Kamra, L. Bülow, J. C. Leo, D. Linke, L. Ye, Angew. Chem. Int. Ed. 2014, 53, 10687-10690; Angew. Chem. 2014, 126, 10863-10866.

[17] a) E. Yilmaz, K. Haupt, K. Mosbach, Angew. Chem. Int. Ed. 2000, 39, 2115-2118; Angew. Chem. 2000, 112, 2178-2181; b) A. Cumbo, B. Lorber, P. F. X. Corvini, W. Meier, P. Shahgaldian, Nat. Commun. 2013, 4, 1503; c) B. A. Menaker, V. Syritski, J. Reut, A. Öpik, V. Horváth, R. E. Gyurcsányi, Adv. Mater. 2009, 21, 2271-2275; d) Z. Bie, Y. Chen, J. Ye, S. Wang, Z. Liu, Angew. Chem. Int. Ed. 2015, 54, 10211-10215; Angew. Chem. 2015, 127, $10349-10353$.

[18] a) Y. Xu, R. D. Marco, A. Shvarey, E. Bakker, Chem. Commun. 2005, 3074-3076; b) M. Pawlak, G. Mistlberger, E. Bakker, Microchim. Acta 2015, 182, 129-137; c) R. E. Gyurcsányi, T. 
Vigassy, E. Pretsch, Chem. Commun. 2003, 2560 -2561; d) Y. Xu, E. Bakker, Langmuir 2009, 25, 568-573; e) M. S. Ozdemir, M. Marczak, H. Bohets, K. Bonroy, D. Roymans, L. Stuyver, K. Vanhoutte, M. Pawlak, E. Bakker, Anal. Chem. 2013, 85, 4770 4776.

[19] a) H. Lee, B. P. Lee, P. B. Messersmith, Nature 2007, 448, 338 341; b) H. Lee, S. M. Dellatore, W. M. Miller, P. B. Messersmith, Science 2007, 318, 426-430.

[20] a) S. H. Yang, S. M. Kang, K. B. Lee, T. D. Chung, H. Lee, I. S. Choi, J. Am. Chem. Soc. 2011, 133, 2795-2797; b) R. Ouyang, J. Lei, H. Ju, Chem. Commun. 2008, 5761-5763; c) W. H. Zhou, C. H. Lu, X. C. Guo, F. R. Chen, H. H. Yang, X. R. Wang, J. Mater. Chem. 2010, 20, 880-883.

[21] X. Wei, X. Li, S. M. Husson, Biomacromolecules 2005, 6, 1113 1121.

[22] a) A. Shvarev, E. Bakker, J. Am. Chem. Soc. 2003, 125, $11192-$ 11193; b) S. Makarychev-Mikhailov, A. Shvarev, E. Bakker, J. Am. Chem. Soc. 2004, 126, 10548-10549; c) G. A. Crespo, M. G. Afshar, E. Bakker, Angew. Chem. Int. Ed. 2012, 51, 12575 12578; Angew. Chem. 2012, 124, 12743-12746.
[23] a) K. L. Gemene, M. E. Meyerhoff, Anal. Chem. 2010, 82, $1612-$ 1615 ; b) J. Lester, T. Chandler, K. L. Gemene, Anal. Chem. 2015, $87,11537-11543$

[24] J. W. Ding, W. Qin, J. Am. Chem. Soc. 2009, 131, 14640-14641.

[25] a) H. S. M. Abd-Rabboh, S. A. Nevins, N. Dürüst, M. E. Meyerhoff, Biosens. Bioelectron. 2003, 18, 229-236; b) J. Yun, M. E. Meyerhoff, Anal. Biochem. 1995, 224, 212-220; c) Y. Chen, J. W. Ding, W. Qin, Bioelectrochemistry 2012, 88, $144-$ 147.

[26] a) A. T. de Souza Liberal, E. A. da Silva Filho, J. O. F. de Morais, D. A. Simões, M. A. de Morais Jr, Lett. Appl. Microbiol. 2005, 40, 19-23; b) L. Granchi, M. Bosco, A. Messini, M. Vincenzini, J. Appl. Microbiol. 1999, 87, 949-956.

Manuscript received: February 21, 2017

Revised manuscript received: April 8, 2017

Version of record online: May 9, 2017 